\section{Chirality of an achiral bent-core nematic mesogen observed in planar and homeotropic cells under certain boundary conditions}

\author{
Yun Jang, ${ }^{a}$ Reshma Balachandran, ${ }^{a}$ Christina Keith, ${ }^{b}$ Anne Lehmann, ${ }^{b}$ Carsten Tschierske ${ }^{b}$ and Jagdish K. Vij ${ }^{* a}$ \\ Received 26th June 2012, Accepted 30th July 2012 \\ DOI: $10.1039 / \mathrm{c} 2 \mathrm{sm} 26483 \mathrm{~h}$
}

It is shown that planar and homeotropic cells under certain boundary conditions containing an achiral bent-core C5 liquid crystalline material show chiral domains of opposite handedness. However such domains are not seen in other members of the homologous series which show cybotactic clusters of larger sizes. The stability of such chiral domains in a planar cell depends not only on temperature but also on the device parameters such as the cell gap and the angle between the rubbing directions on the substrates. The energy difference between the two states with different twist angles measured with respect to the rubbing direction is expressed in terms of the device parameters. The chirality of the material with a pitch length of $9 \mu \mathrm{m}$ is discussed in terms of the molecular symmetry created by the formation of a pair of the two bent-core molecules, which acts as a chiral dopant. The results of the study on C5 are compared with a prediction from a theoretical model with higher order molecular interactions in the bent-core system and it is suggested that the observed phase is a tetrahedratic chiral nematic phase of $D_{2}$ symmetry as recently suggested by [Longa et al., Phys. Rev. E: Stat., Nonlinear, Soft Matter Phys., 2009, 79, 040701].

\section{Introduction}

The chirality of liquid crystals (LCs) has been a topic of enormous interest especially in blue and twist grain boundary (TGB) phases. The chirality arises either from the molecular asymmetry of the LCs or from the formation of supra-structure in the mesophase. ${ }^{1}$ Recently, a number of achiral bent-core liquid crystals (BCLCs) are reported to show chirality in a mesophase. ${ }^{1-4}$ Such a phenomenon of chirality formed from achiral molecules is scientifically intriguing as most commonly the chiral phase with chiral structures arises from the organization of chiral liquid crystalline molecules. Dynamically, standard organic molecules can have an infinite number of different chiral conformations which may exist in fast equilibrium under various experimental conditions. Exceptionally, some molecules, with a large rotational barrier along a covalent bond involved in connecting the two moieties, have a stable chiral structure. These stable chiral conformers are used as ligands in asymmetric synthesis to create new chiral materials. ${ }^{5}$ Flexible achiral BCLCs can also form transient chiral conformations which may lead to chiral nucleation. This is a possible interpretation of the chirality of achiral BCLCs. ${ }^{6}$ In a system consisting of racemized conformers, chiral conformers instantaneously form a homochiral cluster with the same handedness via a self-assembly

${ }^{a}$ Department of Electronic and Electrical Engineering, Trinity College Dublin, University of Dublin, Dublin 2, Ireland. E-mail: jvij@tcd.ie; Fax: +3531677 2442; Tel: +35318961431

${ }^{b}$ Institute of Organic Chemistry, Martin Luther-University HalleWittenberg, D 06120, Germany process. A local chiral phase can thus be formed by additional packing of molecules in such a way that it lowers the free energy. As a result, mostly two domains with opposite handedness are observed or in some cases a single homogeneously chiral domain in the phase ${ }^{3,7}$ is also observed. Recently, a computational study ${ }^{8}$ has shown that chiral superstructures can arise from rigid achiral molecules by self-assembly. A consistent twist sense can also be induced by the chiral dopants in which the chiral nucleation otherwise is not possible. In both interpretations, the chirality of the BCLCs in a mesophase is associated with either local clustering of molecules or via self-assembly of molecules. The presence of cybotactic clusters of smectic-like structures in the nematic phase has been reported by several groups. ${ }^{9-18}$ The materials shown in Fig. 1 are the derivatives of 4-cyanoresorcinol bisbenzoates with different chain lengths in the terminal groups. Dielectric studies show that some of these banana systems with different chain lengths exhibit short-range order. Fig. 2 shows a comparative behavior of the homologous members of this series. The possibility of a short range order in the nematic phase of $\mathrm{C} 5$ cannot be completely excluded (see Fig. 2) compared to the $\mathrm{C} 4$ homologue even though $\mathrm{N}_{\mathrm{CybC}}$ occurs when $n \geq 6$. This indicates that the phase behavior of $\mathrm{C} 5$ in the nematic phase is an



Fig. 1 Chemical structure of 4-cyanoresorcinol bisbenzoates. 


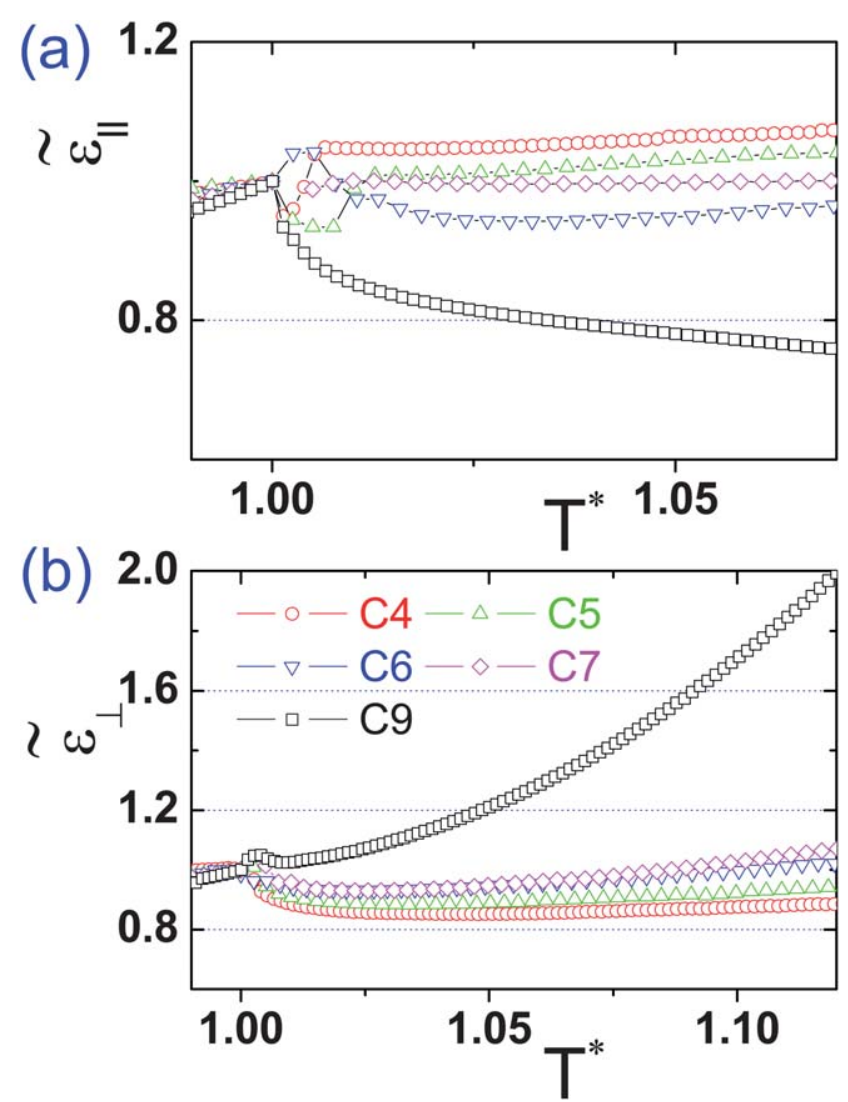

Fig. 2 Comparison of the normalized permittivities $\left(\tilde{\varepsilon}_{\|}\right.$and $\left.\tilde{\varepsilon}_{\perp}\right)$ at a frequency of $1 \mathrm{kHz}$. $T^{*}$ means $T_{\mathrm{c}} / T$. (a) Perpendicular components and (b) parallel components are divided by the value of transition temperature respectively. Here $n_{\mathrm{c}}$ is assumed to be 1.6 and temperature independent befringence $\left(\Delta n_{\mathrm{o}}\right)$ is 0.17 which is measured by the PEM. $n_{\|}$and $n_{\perp}$ are calculated to be 1.71 and 1.54 respectively.

intermediate between $\mathrm{N}$ and $\mathrm{N}_{\mathrm{CybC}}$ (see Table 1). Interestingly, only $\mathrm{C} 5$ in the series of these materials shows chirality in the nematic phase. Therefore it is unclear whether the chirality of $\mathrm{C} 5$ is due to the presence of clusters. Basically BCLCs are considered as racemized conformers, averaged to a V-like molecular shape with $C_{2 v}$ symmetry, which is achiral. A bent shaped molecule of such a symmetry leads to complicated molecular quadrupolar and octupolar interactions. ${ }^{19}$ In a simpler system that comprises two BCMs, combining two molecules yields various symmetries. ${ }^{20,21}$ Such symmetries created through a pairing of the two BCLCs, with nonzero quadrupolar and octupolar interactions, can create additional order parameters such as the biaxiality of mesophase and the chirality via spontaneous symmetry breaking. In this paper, we present the chirality and the phase behavior of a bent-core mesogen (C5 in Table 1).

The chain length of the materials plays a key role in creating a short range order and in forming clusters of a certain size. As shown in Table 1, the nematic phase with smectic $\mathrm{C}$ type nanostructure $\left(\mathrm{N}_{\mathrm{CybC}}\right)$ occurs when $n \geq 6$. The existence of the local nanostructure in the entire nematic phase is supported by the results of the X-ray study with these materials ${ }^{10}$ and by the dielectric studies of $\mathrm{C} 4, \mathrm{C} 7$ and $\mathrm{C} 9 .{ }^{22}$

The dielectric spectra of the bent-core mesogens are recorded as a function of temperature and frequency. The static
Table 1 Transition temperature $\left(T /{ }^{\circ} \mathrm{C}\right)$ and the associated enthalpy values $\left[\Delta H / \mathrm{kJ} \mathrm{mol}^{-1}\right]$ given in square brackets of the 4-cyanoresorcinol bisbenzoates $^{a}$

Comp $n$ Phase transitions

\begin{tabular}{|c|c|c|}
\hline 4 Cr $117[38.4]$ & & (N $107[0.5])$ \\
\hline $5 \mathrm{Cr} 108$ [39.6] & & $\mathrm{N}_{\mathrm{T}}^{*} 108.5[0.5]$ \\
\hline $6 \mathrm{Cr} 98$ [37.1] & & $\mathrm{N}_{\text {CybC }} 101[0.5]$ \\
\hline 7 Cr $96[35.5]$ & $\left(\mathrm{SmC}_{(\mathrm{I})} 25[0.3]\right.$ CybC $\left.41[0.2]\right)$ & $\mathrm{N}_{\text {CybC }} 111[0.7]$ \\
\hline 9 Cr 98[35.1] & $\left(\mathrm{SmC}_{(\mathrm{I})} 50[0.6]\right.$ CybC $\left.58[0.3]\right)$ & $\mathrm{N}_{\text {CybC }} 104[0.8$ \\
\hline
\end{tabular}

${ }^{a}$ Peak temperatures in the first DSC heating curves $\left(10 \mathrm{~K} \mathrm{~min}^{-1}\right)$, monotropic transitions (enclosed by round brackets) were taken from the second heating run; this was easily done because the compounds crystallize slowly and can be supercooled for a prolonged time. Abbreviations: $\mathrm{Cr}=$ crystalline; $\mathrm{I}=$ isotropic phase; $\mathrm{N}=$ nematic phase; $\mathrm{N}_{\mathrm{CybC}}=$ nematic phase having SmC like cybotactic clusters; CybC $=$ mesophase composed of elongated cybotactic clusters; $\mathrm{SmC}_{(\mathrm{I})}$ $=$ synclinic tilted SmC phase; $\mathrm{N}_{\mathrm{T}}^{*}=$ chiral nematic phase with a pair of molecules. Data from Keith et al. ${ }^{10}$

permittivity is calculated by summing up the dielectric strengths of the various processes using Maier and Meier equations:

$$
\begin{gathered}
\varepsilon_{\|}-n_{\|}^{2}=\frac{N h F g_{\|}}{3 \varepsilon_{0} k_{\mathrm{B}} T}\left[\mu_{1}^{2}(1+2 S)+\mu_{\mathrm{t}}^{2}(1-S)\right] \\
\varepsilon_{\perp}-n_{\perp}{ }^{2}=\frac{N h F g_{\perp}}{3 \varepsilon_{0} k_{\mathrm{B}} T}\left[\mu_{1}^{2}(1-S)+\mu_{\mathrm{t}}^{2}\left(1+\frac{1}{2} S\right)\right] \\
\langle\varepsilon\rangle-n^{2}=\frac{N h F_{\mathrm{i}}}{\varepsilon_{0}}\left(\frac{g_{\mathrm{N}} \mu^{2}}{3 k T}\right)
\end{gathered}
$$

Here $S$ is the order parameter. $\mu_{1}=\mu \cos \beta$ and $\mu_{\mathrm{t}}=\mu \sin \beta$ are the longitudinal and transverse components of the molecular dipole moment $(\mu) . \beta$ is the angle the molecular dipole moment makes with respect to the director $\boldsymbol{n} . n_{\|}$and $n_{\perp}$ are the parallel and perpendicular refractive indices respectively. $\frac{N h F g_{\|}}{3 \varepsilon_{0} k_{\mathrm{B}} T}$ and $\frac{N h F g_{\perp}}{3 \varepsilon_{0} k_{\mathrm{B}} T}$ are the multiplying factors for the two permittivities. $N$ is the number density, $\varepsilon_{0}$ is the permittivity of free-space, $k_{\mathrm{B}} T$ is the thermal energy, $F$ and $h$ are the internal field factors for the reaction and the cavity fields. $g_{\|}$and $g_{\perp}$ are the anisotropic Kirkwood correlation factors for the director parallel and perpendicular to the electric field, respectively. $g_{\mathrm{N}}$ is the net dipole correlation factor.

Eqn (1) and (2) give relationships between the measured components of the dielectric permittivity and the molecular dipole moment as a function of $S$. Additionally the number density $N$ (number of molecules per unit volume) must be specified, and correction factors $(g), F$ and $h$ for the internal field evaluated from the same model. While the qualitative behavior of the permittivity can mostly be explained in terms of these equations, they do not provide a quantitative theory of dielectric behavior. For simplicity, we assume $\frac{N h F g_{\mathrm{c}}}{3 \varepsilon_{0} k_{\mathrm{B}} T}\left[\mu_{1}^{2}+\mu_{\mathrm{t}}^{2}\right]$ at the clearing temperature to be unity in the temperature range of the nematic phase. The value of $\frac{N h F g_{\mathrm{c}}}{3 \varepsilon_{0} k_{\mathrm{B}}}\left[\mu_{1}^{2}+\mu_{\mathrm{t}}^{2}\right]$ can be obtained by fitting the permittivity in the temperature range of the isotropic phase. $g_{\mathrm{c}}$ is the Kirkwood correlation factor at the 
clearing temperature. Then the permittivities due to dipole contributions can be normalized by adjusting the value of eqn (4) to eqn (1) and (2).

$$
\begin{gathered}
A=\varepsilon_{\mathrm{c}}{ }^{\prime}-n_{\mathrm{c}}{ }^{2}=\frac{N h F^{2} g_{\mathrm{c}}}{3 \varepsilon_{0} k_{\mathrm{B}} T_{\mathrm{c}}}\left[\mu_{1}{ }^{2}+\mu_{\mathrm{t}}{ }^{2}\right] \\
\tilde{\varepsilon}_{\|}=\frac{\varepsilon_{\|}{ }^{\prime}-n_{\|}{ }^{2}}{\varepsilon_{\mathrm{c}}-n_{\mathrm{c}}{ }^{2}}=\frac{\tilde{g}_{\|}}{T^{*}}\left[1+\left(3 \cos ^{2} \beta-1\right) S\right] \\
\tilde{\varepsilon}_{\perp}=\frac{\varepsilon_{\perp}{ }^{\prime}-n_{\perp}{ }^{2}}{\varepsilon_{\mathrm{c}}-n_{\mathrm{c}}{ }^{2}}=\frac{\tilde{g}_{\perp}}{T^{*}}\left(1+\frac{1-3 \cos ^{2} \beta}{2} S\right)
\end{gathered}
$$

Here $\varepsilon_{\mathrm{c}}{ }^{\prime}$ and $n_{\mathrm{c}}$ are the permittivity and refractive index at the clearing temperature $\left(T_{\mathrm{c}}\right)$, respectively. $\tilde{g}_{\|}$and $\tilde{g}_{\perp}$ are defined as $g / g_{\mathrm{c}}$ and $g_{\perp} / g_{\mathrm{c}}$ respectively. Fig. 2(a) and (b) show the normalized values, $\tilde{\varepsilon}_{\|}$and $\tilde{\varepsilon}_{\perp}$ at a frequency of $1 \mathrm{kHz}$ as a function of $T^{*}\left(T_{\mathrm{c}} / T\right)$ for parallel and perpendicular configurations, respectively. The permittivities at $1 \mathrm{kHz}$ can be regarded as the static value to the extent that the dc conductivity and the relaxations due to end over end rotation are excluded in the spectra. It is clearly seen from Fig. 2(a) and (b) that as the terminal chains get longer from $\mathrm{C} 4$ to C9, $\tilde{\varepsilon}_{\|}$of the materials decreases, while $\tilde{\varepsilon}_{\perp}$ increases in the temperature range of the nematic phase. The parameters $A$ and $\beta$ are independent of the length of the terminal groups, the disagreement among the normalized values in the nematic phase can be interpreted in terms of the $g$-factors. The nematic phase in $\mathrm{C} 4$ is found to be consistent with the M-M model with $g_{\|} \approx g_{\perp} \approx$ $1 .^{22}$ If the anisotropic $g$-factors of $\mathrm{C} 4$ are independent of $S$, the anisotropic correlation factors $\left(g_{\|}\right.$and $\left.g_{\perp}\right)$ of the other materials are affected by the length of the terminal groups. As the terminal groups get longer, $g_{\perp}$ increases and $g_{\|}$decreases. This means that longitudinal dipoles interact in antiferroelectric order, whereas ferroelectric interaction occurs between the transverse dipoles. The behavior immediately after the transition to the nematic phase might follow from the anisotropy of polarizability $(\Delta \alpha)$. Interestingly, $\tilde{\varepsilon}_{\|}$of C6 appears to deviate from the trend of $\tilde{\varepsilon}_{\perp}$ of the other materials (see Fig. 2(a)). Recently, Salter et al. ${ }^{23}$ reported that the flexoelectric coefficient of C6 is greater than that of conventional calamitic LCs. Above all, Fig. 2 indicates that different materials have different anisotropic Kirkwood correlation factors, parallel and normal to the external field, assuming that the order parameter $(S)$ and the angle the molecular dipole moment makes with the director $(\beta)$ are independent of the chain length i.e. the same for different members of the homologous series. This implies that the lateral correlation of the dipoles increases as the chain length increases and this coincides with the results of the X-ray study carried out by Tschierske et al. ${ }^{10}$ The anisotropic Kirkwood correlation factors are interpreted in terms of the size of the cluster, converted then to the number of molecules involved in the cluster. ${ }^{22}$ It is notable that a possibility of the short range order in C5 cannot be excluded from the Kirkwood correlation factors compared to the $\mathrm{C} 4$ homologue. The polarizing microscopy shows that planar cells containing $\mathrm{C} 5$ do not show a dark state as the cell is rotated between the crossed polarizers.

\section{Results}

The transmittance of the cells containing C5 and C6 are compared for different angles between the front and the rear polarizers and by changing the azimuthal angle. The angle between the two polarizers is denoted as $\Theta$. $\Phi$ is the angle between the polarizer and the rubbing direction and is called as the azimuthal angle. Fig. 3(a) and (c) show the transmittance of the cell with C5 in a $10 \mu \mathrm{m}$ cell gap and for C6 in a $5 \mu \mathrm{m}$ cell gap both with planar surface conditions as a function of the wavelength and the azimuthal angle. Fig. 3(b) and (d) show the transmittance when the polarizers are decrossed for C5 and C6, respectively. In a $10 \mu \mathrm{m}$ cell containing $\mathrm{C} 5$, the dark state of a typical planar cell under the condition that rubbing direction is parallel to one of the polarizers cannot be observed as shown Fig. 3(a). Meanwhile, the minimum transmittance as a function of wavelength is dependent on the angle between the polarizers $\Theta$ in Fig. 3(b). Such a behavior of the planar nematic cell with C5 is reminiscent of a twisted nematic cell. Subsequently, we checked the spontaneous twisting of materials with homeotropic anchoring condition. A typical helical structure of chiral nematic LCs is observed as shown in Fig. 4(a) and (b). Interestingly, these results are found only in the cells containing $\mathrm{C} 5$. The cells containing C6 and other compounds of this series do not show chirality up to a cell gap of $35 \mu \mathrm{m}$. In order to measure the pitch length, $P_{1}$, of C5, a Grandjean-Cano wedge cell with a thickness between the two confined glass substrates varying up to $50 \mu \mathrm{m}$ is used. The planar alignment is achieved by using a polymer layer. The cell with planar anchoring shows several disclination lines; these lines are orthogonal to the direction of the increasing layer thickness (assumed as the spacer thickness), shown in Fig. 5. The disclination lines correspond to a change in the twist angle across the layers as $\pi$ and these occur when the cell thickness, $d$ is related to the pitch length. This is expressed as below. ${ }^{24}$

$$
d_{n}=(2 n-1) \frac{P_{1}}{4} ; n=1,2,3, \ldots \ldots \ldots
$$
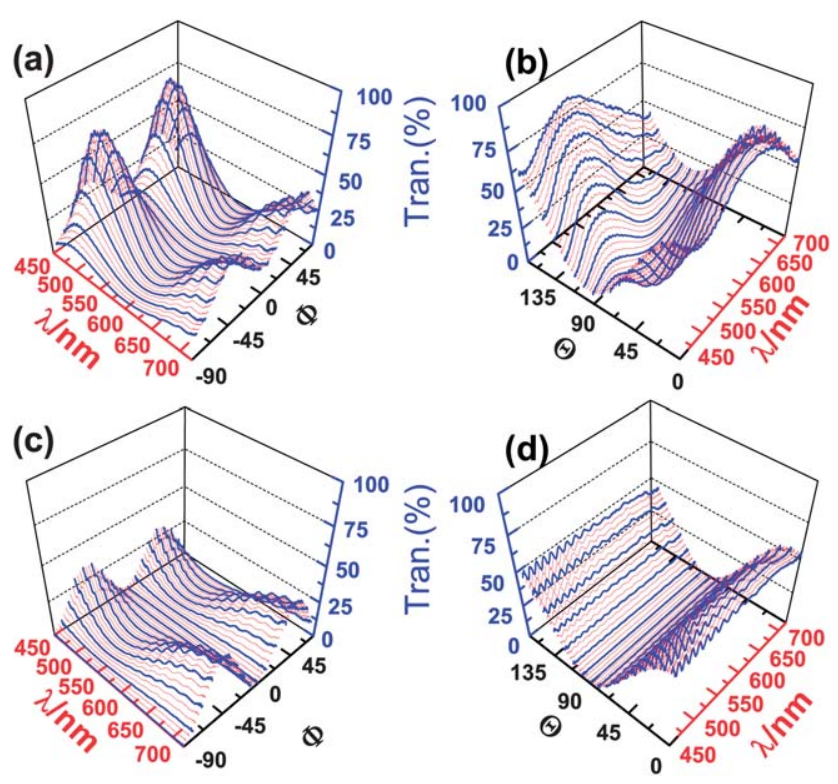

Fig. 3 ( $a$ and $b$ ) are the transmittances of the cell made of $\mathrm{C} 5$ measured for various values of $\Phi$ (the angle between the rubbing direction and one of polarizers) and $\Theta$ (angle between the polarizers), respectively. (c and d) are those for C6, at $\Delta T=5 . \Delta T=\left(T_{\mathrm{c}}-T\right) / K . \Delta T=\left(T_{\mathrm{c}}-T\right) / K$. (c and d) show typical behavior of a planar cell. (a and $b$ ) show those of a twisted cell. 


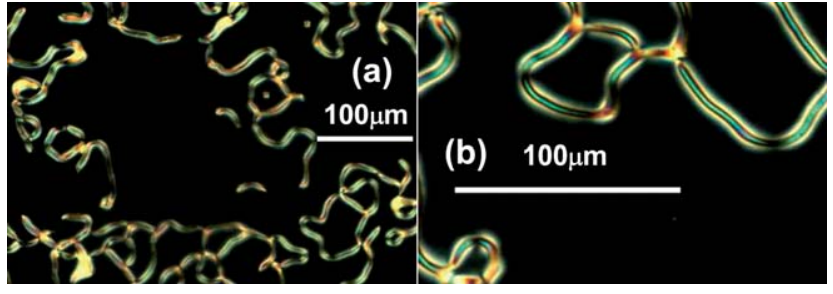

Fig. 4 Fingerprint texture of $\mathrm{C} 5$ under homeotropic anchoring conditions. The cell gap is $8.7 \mu \mathrm{m}$ at $\Delta T=1$. The length of the white bar is $100 \mu \mathrm{m}$.

The distance between the disclination lines gradually decreases as the cell gap increases. This might arise from an uneven bending of one of the substrates. It is very difficult to estimate an accurate structure of the wedge cell due to the gradually changing cell gap. For more accurate determinations of the pitch length, we carried out a qualitative comparative experiment. Fig. 6 shows color comparisons between the second domain $(2 \pi$ twisted) of the wedge cell and a $2 \pi$ twisted cell of $10.1 \mu$ m cell gap under the same experimental conditions. We can decide on a position which shows the same color property dependent on $\Theta$ (the cell gap gradually increases from the left to the right side of the figure). This positional information indicates that the pitch length of $\mathrm{C} 5$ is approximately $9 \mu \mathrm{m}\left(P+\frac{P}{8}=10.1 \mu \mathrm{m}\right)$. Subsequently, a comparison between the first domain and a $\pi$ twisted cell of $5 \mu \mathrm{m}$ cell gap was also carried out. Interestingly, any position with the same color property could not be determined. This might indicate that the $\pi$ twisted cell of $5 \mu \mathrm{m}$ cell gap has the opposite handedness to that of the wedge cell. In general, chiral materials with a pitch length of $200 \mu \mathrm{m}$ are used in many commercial twisted nematic cells to reduce twist degeneracy and the materials with $20 \mu \mathrm{m}$ pitch length are used to induce the higher twist angles found in super twisted nematic cells. ${ }^{24} \mathrm{~A}$ comparison between the pitch length of the commercial materials and that of $\mathrm{C} 5$ indicates that $\mathrm{C} 5$ has quite a strong helical twisting power which is inversely proportional to the pitch length $\left(P_{1}\right)$. Even though we did not measure the twist elastic constant $K_{22}$, we can guess that $\mathrm{C} 5$ has a larger $K_{22}$ than the other elastic constants. This is associated with the pitch length.

It is conceivable that the twisting power could possibly have been induced with a contamination from a chiral impurity for the

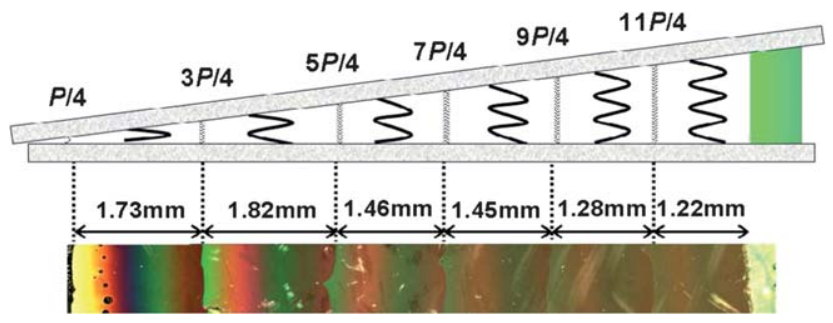

Fig. 5 Due to a limited field of view of the microscope used in the study, each domain is taken individually and combined to exhibit a panoramic view. A $50 \mu \mathrm{m}$ film type spacer was used for the gradually increasing cell gap and the other side was grinded for a flat edge. The spacing of each domain ranges from $1.22 \mathrm{~mm}$ to $1.82 \mathrm{~mm}$. The disclination lines in the wedge cell, so-called Grandjean-Cano wedge cell, correspond to a pitch of $\begin{array}{lllllll}1 & 3 & 5 & 7 & 9 & 11\end{array}$

$\frac{1}{4}, \frac{3}{4}, \frac{5}{4}, \frac{7}{4}, \frac{1}{4}, \frac{11}{4}$ sequentially from left to right in the figure.

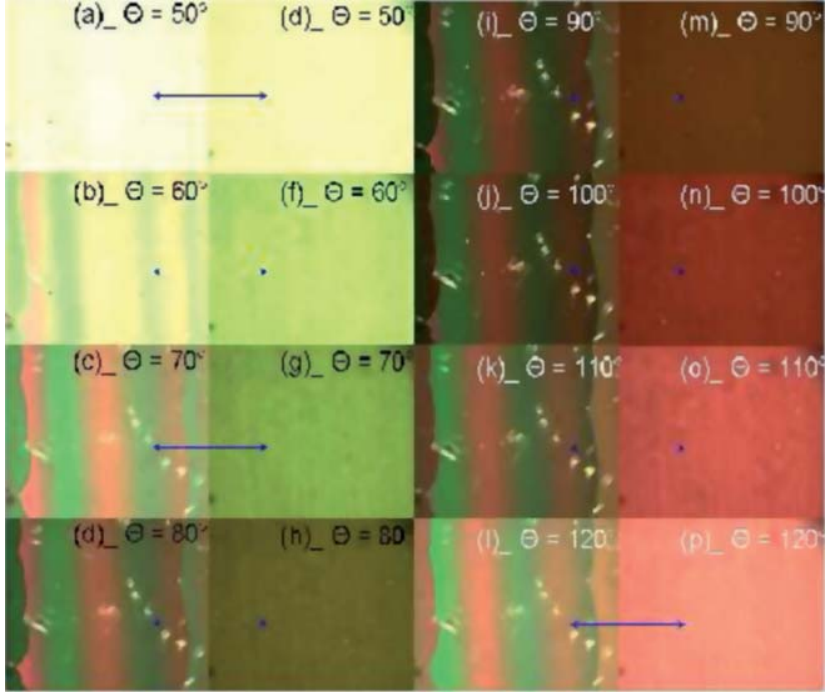

Fig. 6 The colors of the $2^{\text {nd }}$ domain for various $\Theta$ s (angles between the two polarizers) are compared with a planar cell of $10.1 \mu \mathrm{m}$ cell gap at $\Delta T=25 . \Delta T=\left(T_{\mathrm{c}}-T\right) / K$. (a-d), (i-l) were taken from the second domain of the wedge cell with an analyzer rotated, $(\mathrm{e}-\mathrm{h}),(\mathrm{m}-\mathrm{p})$ are taken from the planar cell of $10.1 \mu \mathrm{m}$ under the same conditions, respectively. $\Theta$ means the angle between the polarizers, the arrows indicate the position with same color, in which two cells have the same cell gap.

case when a homochiral phase is observed. If we are able to make two cells with opposite handedness, it would be a proof for excluding the chiral contamination. However, under practical experimental conditions, it was very difficult to reproduce cells with the same handedness and the cell gap. In order to exclude the possibility of contamination, we investigated the chirality with two C5 samples, synthesized separately. One of the synthetic routes is based on using pentanoic acid and the second using pentanol/pentylbromide. Both samples show compatible chirality. Therefore there is no special reason as to why there should be a chiral impurity. Nevertheless we cannot completely exclude traces of the chiral material that could have been present in the starting materials, since the synthesis started from the alkanoic acids. However the probability of chiral contamination is not any higher for C5 compared to the other members of the homologous series such as C4, C6, C7 and C9. It is known that in an ideal planar cell, a right handed twisted domain has exactly the same free energy as the left handed one which means that the two states are degenerate. As a consequence, the two domains with opposite handedness can coexist $^{3,7}$ in the mesophase. However, the rubbing direction of one of the substrates used in the cell can most likely deviate from the second rubbing direction in the device. In such a case, due to a deviation angle $\left(\psi_{\mathrm{R}}\right)$ between the two rubbing directions in a planar cell, the twist angle of one state is different from the other. The twist energy $F_{\mathrm{t}}$ per unit volume of a layer of thickness $d$ and the twist angle $\phi$ can be found from the continuum equation:

$$
\begin{gathered}
F_{\mathrm{t}}=\frac{K_{22}}{2}\left(\frac{\phi}{d}-\frac{2 \pi}{P}\right)^{2} \\
F_{\mathrm{R}}=\frac{K_{22}}{2}\left(\frac{\phi+\psi_{\mathrm{R}}}{\mathrm{d}}-\frac{2 \pi}{P}\right)^{2}
\end{gathered}
$$




$$
\begin{gathered}
F_{\mathrm{L}}=\frac{K_{22}}{2}\left(\frac{\phi-\psi_{\mathrm{R}}}{d}-\frac{2 \pi}{P}\right)^{2} \\
\Delta F_{\mathrm{t}}=\frac{2 K_{22} \psi_{\mathrm{R}}}{d}\left(\frac{\phi}{d}-\frac{2 \pi}{P}\right)
\end{gathered}
$$

$\Delta F_{\mathrm{t}}$ is the energy difference between $F_{\mathrm{R}}$ and $F_{\mathrm{L}}$. When the deviation angle between the rubbing direction on each glass plate $\psi_{\mathrm{R}}$ is taken into consideration, the energy difference $\Delta F_{\mathrm{t}}$ in the nematic phase between the two states of opposite handedness can be expressed in terms of eqn (11). $\Delta F_{\mathrm{t}}$ is dependent on the cell gap $(d)$, the deviation angle $\left(\psi_{\mathrm{R}}\right)$ and $K_{22}$. In a wedge cell different domains are observed while the sample is cooled from the isotropic to the nematic phase. In a part of the cell with a smaller cell gap, we may get homochiral domains that can be stabilized over a shorter period of time. Meanwhile in a thicker part of the cell, it takes a considerable time for the domains to get stabilized. Besides, as the energy difference between the domains of opposite handedness is small, it is extremely difficult to distinguish one domain from the other with opposite handedness by rotation of an analyzer using the method of color comparisons. The color change brought about by a rotation of the analyzer is too insensitive to confirm the handedness. A $2 \pi$ twisted cell was made with a cell gap of $8.2 \mu \mathrm{m}$. Fig. 7(a) shows a few domains immediately after the transition to the nematic phase. With the passage of time, the two domains merge into a single one as seen in Fig. 7(b). Two of the domains have opposite handedness. This is confirmed by decrossing the polarizers in the opposite direction as shown in Fig. 7(c) and (d). If the twist of the directors were to be induced by a chiral impurity, the domain with one chirality can only be observed on the transition to the nematic phase. Therefore the presence of the domain with opposite handedness is incompatible with an explanation of a sample contamination. Recently, Kim et al. reported a nematic phase $\left(\mathrm{N}_{\mathrm{T}}^{*}\right)^{25}$ to appear along with an earlier discovered isotropic nematic phase $\left(\mathrm{N}_{\mathrm{T}}\right)$ of a BCLC. ${ }^{26}$ The ferrocene derivatives with the free rotation of the

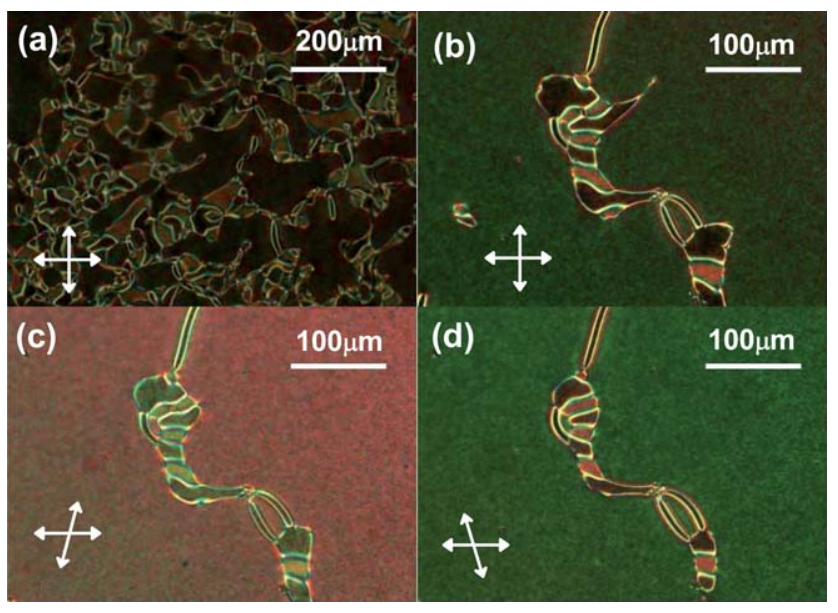

Fig. 7 The domains with opposite chirality coexist over a very short time and merge into one most stable domain of a single handedness in a planar anchoring condition. (a) Taken 15 seconds after transition to the nematic phase at a cooling rate of $1^{\circ} \mathrm{s}^{-1}$ at $\Delta T=11 \mathrm{~K},(\mathrm{~b}-\mathrm{d})$ taken subsequently, between the crossed and de-crossed polarizers, respectively. cyclopentadienyl rings can adopt chiral conformations and these can be interlocked and stabilized with a coupling from the neighboring conformers of the same handedness into tetrahedral dimers. The symmetry breaking arises from the interlocked conformers with the same chirality and $\left(\mathrm{N}_{\mathrm{T}}^{*}\right)$ is generated as well as a chiral smectic phase. In the case of BCMs, such an interlocking through transient chiral conformers is possible under specific conditions that the transitional freedom of LCs is limited to the nearest neighbors where the two bent-core molecules form a tetrahedric dimer. According to our previous work, C5 forms a nematic phase whose nature is intermediate between the usual nematic phase and the $\mathrm{N}_{\mathrm{CybC}}$ phase. The small angle scattering in the X-ray diffraction pattern has been interpreted in terms of the clusters. A weak small angle scattering can be interpreted as an indication of a nematic phase composed of clusters when the number of molecules participating is small. Within a nematic phase, some of the molecules form a simple interlocked cluster that is composed of two bent-core molecules and these behave like a chiral dopant. The other molecules with longer terminal chains build up into larger clusters. These behave like a biaxial particle in a uniaxial nematic phase referred to in the cluster model. For C5, there is the lateral correlation observed by the dielectric measurement (see Fig. 2). This indicates the presence of a certain short-range correlation in the nematic phase.

The most plausible scenario considered here is a coupling of the two BCLCs to form the simplest cluster (see Fig. 8). A preliminary theoretical interpretation of such a system has been given at macroscopic and microscopic scales. ${ }^{\text {20,21,27,28 }}$ According to these theoretical models, such a pairing can form the simplest cluster which can lead to not only the usual nematic phase but also new types of nematic phases such as the biaxial nematic phase $\left(\mathrm{N}_{\mathrm{b}}\right)$, tetrahedratic nematic phase $\left(\mathrm{N}_{\mathrm{T}}\right)$ and the chiral tetrahedratic nematic phase $\left(\mathrm{N}_{\mathrm{T}}^{*}\right)$. They can exist through higher order molecular interactions (quadrupolar, octupolar) in the condensed phase. According to the computational study carried out by Longa et al., ${ }^{21}$ the phase transition from the isotropic to $\mathrm{N}_{\mathrm{T}}^{*}$ phase is not allowed except through the Landau point where all possible phases can coexist under a specific condition. In order to construct the phase diagram, we investigated the transition behavior of the material under extreme slow cooling. Fig. 9

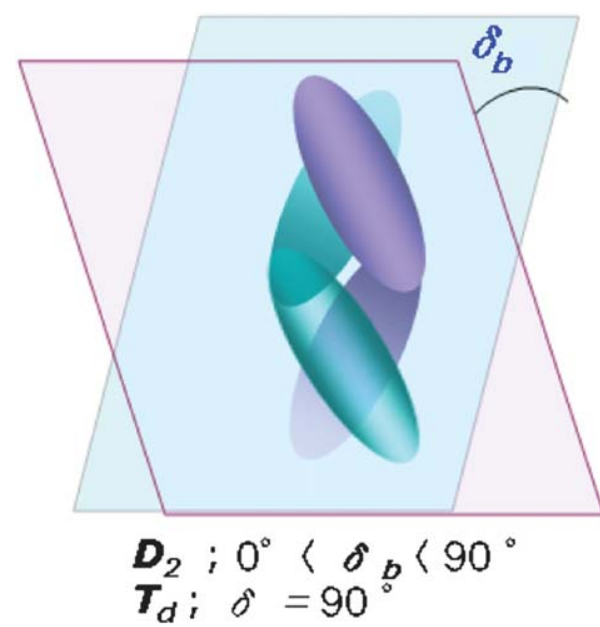

Fig. 8 Formation of $\mathrm{N}_{\mathrm{T}}^{*}$ when $0<\delta_{\mathrm{b}}<\frac{\pi}{2}$. 


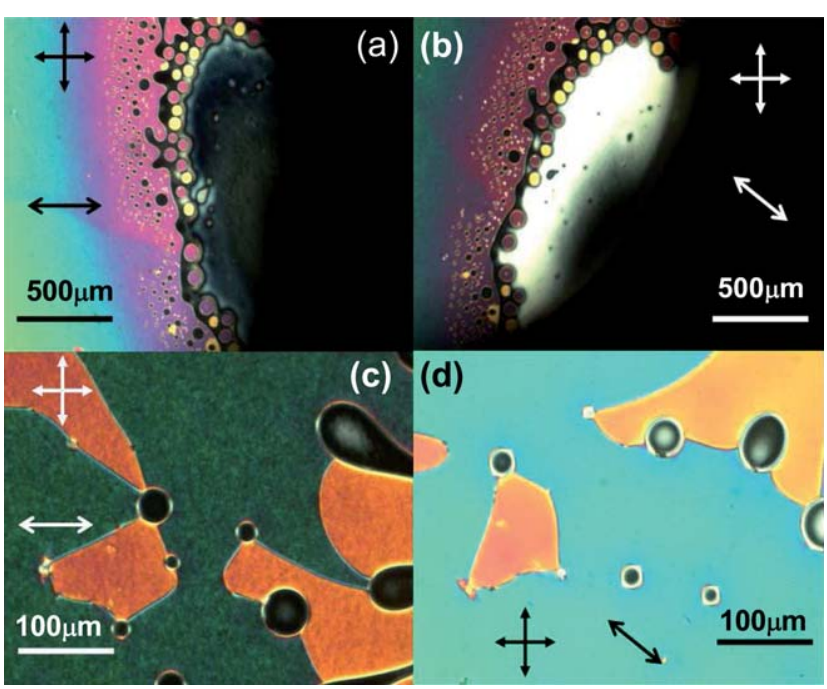

Fig. 9 Transition behavior of $\mathrm{C} 5$ on slow cooling at a rate of $0.5^{\circ}$ per hour.

shows four optically different domains. The left side of the figures is at a slightly lower temperature than the right side due to a temperature gradient in the cell. This is shown by a gradual change in the color of the major domains on the left side of Fig. 9(a) and (b). Single and twin arrows indicated on the figures denote the direction of rubbing and of the polarizers, respectively. Black regions on the right side of Fig. 9(a) and (b) are the non-transparent parts of the sample holder, and the white domain in Fig. 9(b) is the uniaxial nematic phase with the planar anchoring condition. This is confirmed by rotating the stage of the microscope (see Fig. 9(a)). A bright big domain on the left side of Fig. 9(a) and (b) is assigned to the chiral nematic phase. Between the two main domains, domains with black and yellow colors are observed. The latter appears to be the chiral nematic phase with either a different handedness or helicity to the bright domain and the former is optically isotropic under planar anchoring condition, confirmed by a rotation of the stage (Fig. 9(c) and (d)). This optically isotropic nematic phase can be interpreted as a tetrahedratic nematic phase $\left(\mathrm{N}_{\mathrm{T}}\right)$. On the basis of the temperature gradient in the cell, the phase transition of $\mathrm{C} 5$ can be assigned as follows: isotropic, uniaxial nematic, $\mathrm{N}_{\mathrm{T}}, \mathrm{N}_{\mathrm{T}}^{*}$ phases seen upon cooling optically. This result is consistent with the theoretical prediction of Longa et al. ${ }^{21}$ Interestingly, a clear phase boundary is not observed between the uniaxial nematic and $\mathrm{N}_{\mathrm{T}}^{*}$ in Fig. 9. The microscopic structure of $\mathrm{C} 5$ in the nematic phase is still elusive. However the macroscopic behavior of $\mathrm{C} 5$ is well explained with the model of a possible tetrahedric dimer consisting of two molecules.

\section{Conclusion}

It has been shown that planar and homeotropic cells under certain boundary conditions containing an achiral bent-core $\mathrm{C} 5$ liquid crystalline material show chirality. In terms of the phase behavior, the nematic phase of C5 is intermediate between the usual nematic phase and the cybotactic nematic phase. Even though short-range correlation between the molecules of C5 is not as strong as for the molecules with longer terminal groups, C5 shows a considerable twisting power in the entire nematic phase. It is interesting that the chirality is not directly proportional to the formation of the clusters in the nematic phase. The stability of such chiral domains in a planar cell depends not only on temperature but also on the device parameters such as the cell gap and the relative directions of rubbing on the substrates. The energy difference between the two states with different twist angles between the rubbing directions is expressed in terms of the device parameters. The chirality of C5 with a pitch length of $9 \mu \mathrm{m}$ is discussed in terms of the molecular symmetry created by the formation of a pair of two bent-core molecules. Such a pairing of molecules acts as a chiral dopant. The experimental results on the device containing the material are compared with a prediction from a theoretical model ${ }^{9}$ with higher order molecular interactions in the bent-core system and it is suggested that the observed nematic phase is the tetrahedratic chiral nematic phase of $\mathrm{D}_{2}$ symmetry as recently predicted by Longa et $a .^{21}$

\section{Acknowledgements}

Work was carried out under the FP7 EU Bind project (Grant No. 216025). One of the authors (RB) thanks the IRCSET for funding research studentship.

\section{References}

1 R. A. Reddy and C. Tschierske, J. Mater. Chem., 2006, 16, 907.

2 G. Pelzl, A. Eremin, S. Diele, H. Kresse and W. Weissflog, J. Mater. Chem., 2002, 12, 2591; M. Hird, J. W. Goodby, N. Gough and K. J. Toyne, J. Mater. Chem., 2001, 11, 2732; T. Niori, J. Yamamoto and H. Yokoyama, Mol. Cryst. Liq. Cryst., 2004, 409, 475 .

3 V. Gortz and J. W. Goodby, Chem. Commun., 2005, 3262.

4 H. Takezoe and Y. Takanishi, Jpn. J. Appl. Phys., 2006, 45, 597.

5 R. Noyori, I. Tomino and M. Nishizawa, J. Am. Chem. Soc., 1979, 101, 5843.

6 D. J. Earl, M. A. Osipov, H. Takezoe, Y. Takanishi and M. R. Wilson, Phys. Rev. E: Stat., Nonlinear, Soft Matter Phys., 2005, 71, 021706.

7 P. S. Salter, P. W. Benzie, R. A. Reddy, C. Tschierske, S. J. Elston and E. P. Raynes, Phys. Rev. E: Stat., Nonlinear, Soft Matter Phys., 2009, 80, 031701.

8 F. Yan, C. A. Hixson and D. J. Earl, Phys. Rev. Lett., 2008, 101, 157801.

9 C. Tschierske and D. J. Photinos, J. Mater. Chem., 2010, 20, 4263.

10 C. Keith, A. Lehmann, U. Baumeister, M. Prehm and C. Tschierske, Soft Matter, 2010, 6, 1704.

11 V. Görtz, C. Southern, N. W. Roberts, H. F. Gleeson and J. W. Goodby, Soft Matter, 2009, 5, 463.

12 S. H. Hong, R. Verduzco, J. C. Williams, R. J. Twieg, E. DiMasi, R. Pindak, A. Jakli, J. T. Gleeson and S. Sprunt, Soft Matter, 2010, 6, 4819.

13 S. H. Hong, J. C. Williams, R. J. Twieg, A. Jákli, J. Gleeson, S. Sprunt and B. Ellman, Phys. Rev. E: Stat., Nonlinear, Soft Matter Phys, 2010, 82, 041710 .

14 O. Francescangeli, V. Stanic, S. Torgova, A. Strigazzi, N. Scaramuzza, C. Ferrero, I. P. Dolbnya, T. M. Weiss, R. Berardi, L. Muccioli, S. Orlandi and C. Zannoni, Adv. Funct. Mater., 2009, 19, 2592.

15 S. Droulias, A. Vanakaras and D. Photinos, Liq. Cryst., 2010, 37, 969.

16 S. D. Peroukidis, A. G. Vanakaras and D. J. Photinos, Phys. Rev. E: Stat., Nonlinear, Soft Matter Phys., 2011, 84, 010702.

17 O. Francescangeli and E. T. Samulski, Soft Matter, 2010, 6, 2413.

18 O. Francescangeli, F. Vita, C. Ferrero, T. Dingemans and E. T. Samulski, Soft Matter, 2011, 7, 895.

19 M. A. Bates and G. R. Luckhurst, Phys. Rev. E: Stat., Nonlinear, Soft Matter Phys., 2005, 72, 051702. 
20 T. C. Lubensky and L. Radzihovsky, Phys. Rev. E: Stat., Nonlinear, Soft Matter Phys., 2002, 66, 031704.

21 L. Longa, G. Pajak and T. Wydro, Phys. Rev. E: Stat., Nonlinear, Soft Matter Phys., 2009, 79, 040701.

22 Y. Jang, V. P. Panov, A. Kocot, A. Lehmann, C. Tschierske and J. K. Vij, Phys. Rev. E: Stat., Nonlinear, Soft Matter Phys., 2011, 84, 060701(R).

23 P. S. Salter, C. Tschierske, S. J. Elston and E. P. Raynes, Phys. Rev. E. Stat., Nonlinear, Soft Matter Phys., 2011, 84, 031708.

24 E. P. Raynes, Liq. Cryst., 2006, 33, 1215.
25 E.-H. Kim, O. N. Kadkin, S.-Y. Kim and M.-G. Choi, Eur. J. Inorg. Chem., 2011, 2011, 2933.

26 D. Wiant, K. Neupane, S. Sharma, J. T. Gleeson, S. Sprunt, A. Jákli, N. Pradhan and G. Iannacchione, Phys. Rev. E: Stat., Nonlinear, Soft Matter Phys., 2008, 77, 061701.

27 L. G. Fel, Phys. Rev. E: Stat. Phys., Plasmas, Fluids, Relat. Interdiscip. Top., 1995, 52, 702.

28 H. Brand and H. Pleiner, Eur. Phys. J. E: Soft Matter Biol. Phys., 2010, 31, 37. 